\title{
Theoretical and experimental investigation of low-volgage and low-loss 25-Gbps Si photonic crystal slow light Mach-Zehnder modulators with interleaved p/n junction
}

\author{
Yosuke Terada, Hiroyuki Ito, Hong C. Nguyen and Toshihiko Baba*
}

Department of Electrical and Computer Engineering, Yokohama National University, Yokohama, Japan

\author{
Edited by: \\ Kensuke Ogawa, Fujikura \\ Ltd., Japan \\ Reviewed by: \\ Satoshi Iwamoto, The University of \\ Tokyo, Japan \\ Takasumi Tanabe, Keio \\ University, Japan \\ *Correspondence: \\ Toshihiko Baba, Department of \\ Electrical and Computer \\ Engineering, Yokohama National \\ University, 79-5 Tokiwadai, \\ Hodogayaku, Yokohama, Kanagawa \\ 240-8501, Japan \\ e-mail: baba@ynu.ac.jp
}

In this study, we investigated the performance of Si lattice-shifted photonic crystal waveguide (LSPCW) Mach-Zehnder modulators theoretically and experimentally. The LSPCW increases the phase shift in modulator to 2.3-2.5 times higher, which allows for size reduction and high performance. On-chip passive loss was reduced to less than $5 \mathrm{~dB}$ by optimizing each component. We obtained $25 \mathrm{Gbps}$ clear open eye and $3 \mathrm{~dB}$ extinction ratio at a drive voltage of $1.5-1.75 \mathrm{~V}$ for $200 \mu \mathrm{m}$ phase shifter with linear $\mathrm{p} / \mathrm{n}$ junction when we added a modulation loss of $7 \mathrm{~dB}$. This modulation loss was reduced to $0.8 \mathrm{~dB}$, maintaining other performance, by employing interleaved $\mathrm{p} / \mathrm{n}$ junction and optimizing doping concentrations.

Keywords: silicon photonics, modulator, photonic crystal waveguides, optoelectronics, slow light

\section{INTRODUCTION}

Si photonics, which can produce highly integrated photonic circuits using CMOS-compatible process, has been developed extensively toward optical interconnects [1]. Since external light sources are used in current $\mathrm{Si}$ photonics, Si modulators based on carrier plasma dispersion (CPD) [2-20], electro-absorption $[21,22]$, or so are necessary. The CPD in $\mathrm{p} / \mathrm{n}$ diode structures is practically used in commercial modulators for optical interconnects because of the easy fabrication of the $\mathrm{p} / \mathrm{n}$ junction and operation over the wide range of wavelength and temperature, which is crucial in optical interconnects. CPD is generated by carrier injection or depletion. The carrier injection gives a larger refractive index change, and its $3-\mathrm{dB}$ cutoff frequency is $\sim 1 \mathrm{GHz}$ or smaller [2] and preemphasized signals are necessary for higherspeed operation [3-5]. Therefore, the carrier depletion that can respond at higher speeds is often employed although the index change is smaller [6-19]. Using resonance effects such as that in microrings is one of the methods that compensate the small index change, but its narrow band hampers to use over the wide range of temperature $[4,6,7]$. On the other hand, Mach-Zehnder (MZ) interferometer modulators have broad band, allowing wide temperature range operation [2, 3, 5, 8-19]. However, their device size is usually of the millimeter-order $[7-10,12-14]$ and drive voltage is higher than several volts [9-19], which cannot meet the requirements for optical interconnects. To solve this constraint, we have studied slow light with a low group velocity $v_{\mathrm{g}}$ [22-24]. Slow light accelerates the efficiency of MZ modulators in proportion to the group index $n_{\mathrm{g}}=c / v_{\mathrm{g}}$, where $c$ is the light velocity in vacuum. Silica-cladded Si photonic crystal waveguide produces slow light with a typical $n_{\mathrm{g}} \sim 20$ [16] and can be fabricated easily by CMOS-compatible process [23]. We have proposed and demonstrated Si MZ modulators incorporating this waveguide as phase shifters. First, $10 \mathrm{Gbps}$ operation was observed at carrier injection $[5,15]$ and carrier depletion [15] in 200- $\mu \mathrm{m}$-long devices. The preliminary operation at $25-40 \mathrm{Gbps}$ was also obtained in sub-100- $\mu \mathrm{m}$ devices [16]. The operation over the wide range of wavelength $(\Delta \lambda=16.9 \mathrm{~nm})$ and temperature $(\Delta \mathrm{T}=105 \mathrm{~K})$ is confirmed by employing lowdispersion slow light in lattice-shifted photonic crystal waveguide (LSPCW) [17].

In this paper, we discuss the balancing of various performance parameters of the Si LSPCW MZ modulator and improvement of $\mathrm{p} / \mathrm{n}$ junction, which has not been studied so far for this modulator. In Section Requirements, we generally discuss the requirements for Si MZ modulators and interrelation between performance parameters. In Section Calculations, we explain the detailed structure of the modulator in this study. We quantify slow light enhancement by calculating the CPD-induced index change and phase shift of light, and show a guideline for optimization. We also calculate the electrical resistance and junction capacitance, giving the RC time constant, and discuss how it is related with other performance. In Section Fabrication and measurement, we present the fabrication and measurement results, and demonstrate $10-25$ Gbps operations in $90-200 \mu \mathrm{m}$ devices and low-loss operation by the improved $\mathrm{p} / \mathrm{n}$ junction and doping concentrations.

\section{REOUIREMENTS}

In general, the normalized output intensity $I[\mathrm{~dB}]$ of $\mathrm{MZ}$ modulators is given for the optical phase $\phi$ by 


$$
I=10[\log (1+\cos \phi)]-10 \log 2
$$

and is depicted by the solid line in Figure 1. Here the intensity is normalized by that at $\phi=0$, which actually gives the on-chip passive loss $P L$ without modulation. Setting phase operating point $\phi_{1}$ and adding the phase shift $\Delta \phi$, output light is modulated with the extinction ratio $E R$, while the modulation loss $M L$ is added according to $\phi_{1}$. ER and $M L$ are expressed as follows:

$$
\begin{aligned}
E R & =10\left[\log \left(1+\cos \phi_{1}\right)-\log \left(1+\cos \left(\phi_{1}+\Delta \phi\right)\right)\right], \\
M L & =10\left[\log 2-\log \left(1+\cos \phi_{1}\right)\right] .
\end{aligned}
$$

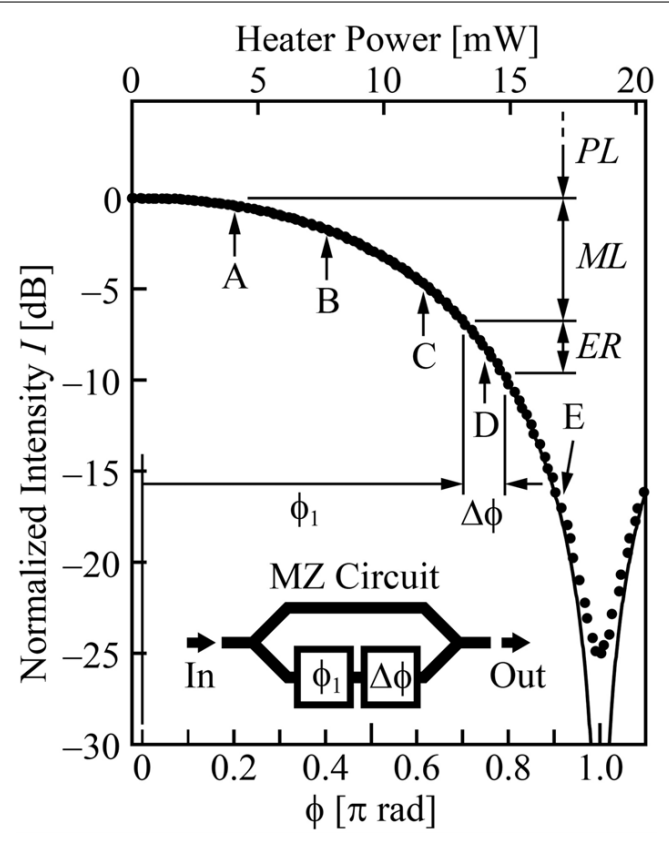

FIGURE 1 | Intensity response (solid line) and measured ones (closed circles) as a function of the phase shift of the MZ modulator and corresponding heater power in phase tuner. The intensity is normalized by that at $\phi_{1}=0$.
From these equations, we obtain

$$
M L=10\left[\log 2-\log \left\{1+\frac{\left(\begin{array}{l}
-\left(1-10^{0.1 E R}\right)\left(1-10^{0.1 E R} \cos \Delta \phi\right) \\
+\sqrt{2 \cdot 10^{0.3 E R}}(1-\cos \Delta \phi) \sqrt{1+\cos \Delta \phi}
\end{array}\right)}{10^{0.2 E R}-2 \cdot 10^{0.1 E R} \cos \Delta \phi+1}\right\}\right]
$$

As $\phi$ approaches to $\pi,|d I / d \phi|=10 \sin \phi /(1+\cos \phi)$ increases, and a large $E R$ is obtained even with small $\Delta \phi$. However, the $M L$ increases simultaneously, resulting in low $\mathrm{S} / \mathrm{N}$. To meet the requirements of optical interconnects, sufficient $E R$ must be obtained with suppressing the total loss $P L+M L$. One target value acceptable may be $P L+M L<10 \mathrm{~dB}$, but $P L+M L \leq 5 \mathrm{~dB}$ will be demanded finally for loss penalty. In view of the operating voltage of high-speed CMOS driver circuits, the drive voltage of the modulator $V_{\mathrm{pp}}$ should be $1.5 \mathrm{~V}$ or lower. The $E R$ of $3 \mathrm{~dB}$ or higher is necessary for discriminating digital data. In addition, the data bitrate $B R \geq 25$ Gbps achieving a $100 \mathrm{Gbps}$ throughput by preparing four traffic channels, phase shifter length $L<$ $1 \mathrm{~mm}$ fitting into a small footprint of optical transceivers and optical interposers, and wavelength range $\Delta \lambda>10 \mathrm{~nm}$ equivalent to the temperature range $\Delta T>100 \mathrm{~K}$ and so on will be expected.

Table 1 compares the performance (except for $\Delta \lambda$ and $\Delta T$ ) of Si MZ modulators based on rib waveguides and LSPCW [8-14, 17]. Rib waveguide modulators satisfy the requirements for $B R$ and $E R$, and not for $L, P L, M L$, and/or $V_{\mathrm{pp}}$. The previous performance of LSPCW modulators is similar, except for $L$. In both modulators, larger $\Delta \phi$ is necessary for satisfying all the requirements. It is obtainable by increasing $n_{\mathrm{g}}, L$, and $V_{\mathrm{pp}}$. Here, $V_{\mathrm{pp}}$ must rather be reduced since $V_{\mathrm{pp}}$ in previous studies are too high. The typical value of $n_{\mathrm{g}}$ is $\sim 20$ (22 is measured in this study, as shown in Section Fabrication and measurement) in the third-row-shifted LSPCW [16]. Although we can increase this value by modifying the structure, $\Delta \lambda$ will be narrowed due to the $n_{\mathrm{g}} \Delta \lambda$ product constraint of slow light and the waveguide loss will be increased by larger $n_{\mathrm{g}}$. We may increase $L$ of the LSPCW since it is already much smaller than the requirement,

\begin{tabular}{|c|c|c|c|c|c|c|c|c|}
\hline Authors (Group) [Ref.] & Waveguide & Junction & $L[\mathrm{~mm}]$ & $P L[\mathrm{~dB}]$ & $M L[\mathrm{~dB}]$ & $V_{p p}[V]$ & $B R$ [Gbps] & $E R[\mathrm{~dB}]$ \\
\hline Ding et al. (University of Dalaware) [8] & Rib & Linear & 3.0 & 6.2 & 1.4 & 1.6 & 40 & 3.1 \\
\hline Yang et al. (CAS) [9] & Rib & Linear & 2.0 & 3.8 & $1.5-2.5$ & 6.0 & 40 & 4.9-6.4 \\
\hline Xu et al. (CAS) [10] & Rib & Interleaved & 1.0 & 3.8 & 6.0 & 3.5 & 40 & 4.1 \\
\hline Kim et al. (ETRI) [11] & Rib & Vertical & 0.1 & 3.0 & 9.3 & 2.5 & 40 & 6.1 \\
\hline Morini et al. (University of Paris-sud) [12] & Rib & Interleaved & 0.95 & 3.5 & 0.5 & 7.0 & 40 & 7.9 \\
\hline Xiao et al. (CAS) [13] & Rib & Linear & 0.75 & 1.9 & 1.5 & 6.5 & 50 & 3.9 \\
\hline Thomson et al. (University of Surrey) [14] & Rib & Linear & 1.0 & 4.2 & 3.2 & 6.0 & 50 & 3.1 \\
\hline Nguyen et al. (YNU) [17] & LSPCW & Linear & 0.2 & $\sim 7$ & 1.1 & 4.7 & 10 & 5.8 \\
\hline Nguyen et al. (YNU) [17] & LSPCW & Linear & 0.09 & N/A & $\mathrm{N} / \mathrm{A}$ & 3.6 & 25 & 2.0 \\
\hline This study & LSPCW & Interleaved & 0.2 & 5 & 0.8 & 1.5 & $10-25$ & 3.0 \\
\hline
\end{tabular}
but it will also increase the waveguide loss. Therefore, we fix these parameters in this paper. Other methods considered for larger $\Delta \phi$

Table 1 | Comparison of performances of modulators.

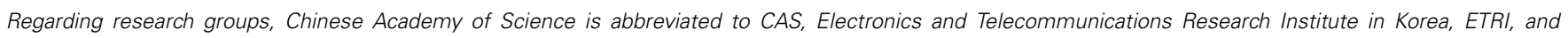
Yokohama National University, YNU. 
are employing interleaved $\mathrm{p} / \mathrm{n}$ junction $[7,10,18,19,25]$ and increasing doping concentration. The former increases the overlap between the depletion layer and waveguide mode. The latter is expected to increase the quantity of the index change within the limits that the free carrier absorption does not affect severely [26].

\section{CALCULATIONS STRUCTURE}

Figure 2 shows the structure of the LSPCW modulator in this study. It consists of two LSPCW phase shifters with $\mathrm{p} / \mathrm{n}$ junctions at the waveguide center, thermo-optic phase tuners with TiN heaters, and MZ circuit of Si wires. We compare linear and interleaved $\mathrm{p} / \mathrm{n}$ junction. The phase tuner in our previous studies was formed at the Si wire. In this study, it is located at the LSPCW so that the slow light enhancement is also expected not only for the phase shifter but also for the phase tuner. Structural

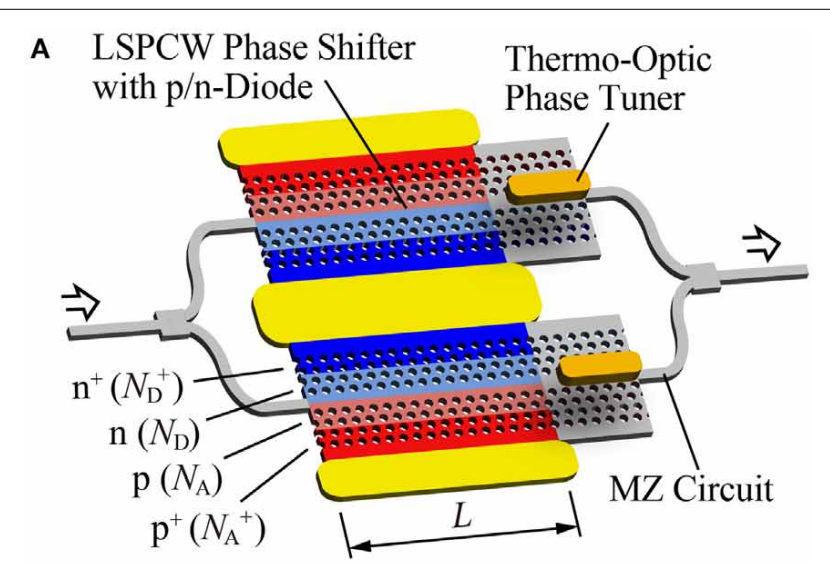

B $\quad$ Linear Junction (\#1) Lattice Shift $s$
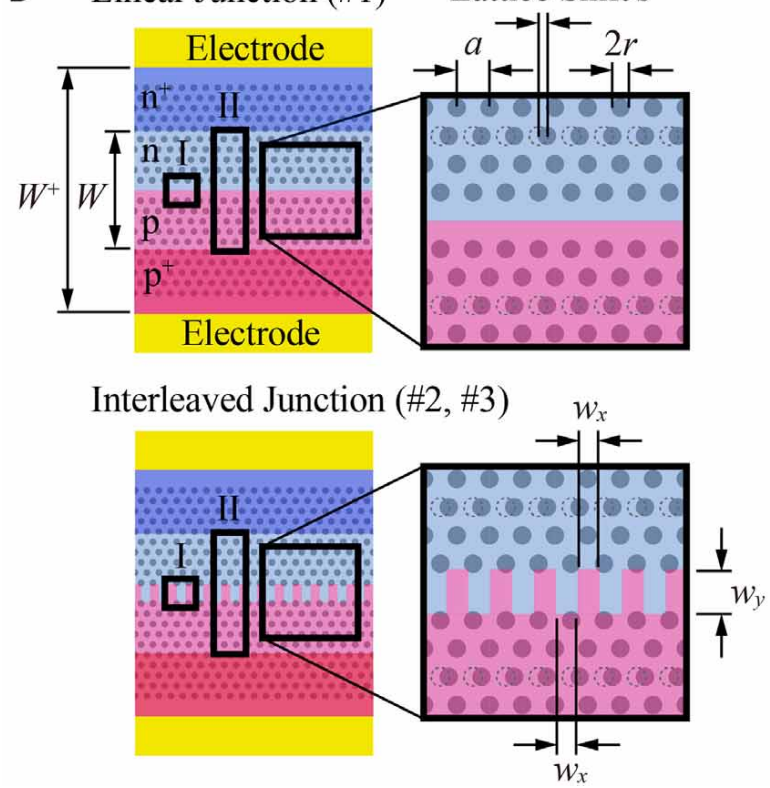

FIGURE 2 | Schematic of Si LSPCW MZ modulator. (A) Total view. (B) Two types of $\mathrm{p} / \mathrm{n}$ junction. I and II correspond to models in Figures 3, 4, respectively. parameters we can design are $L$, LSPCW's lattice constant $a$, hole diameter $2 r$ and lattice shift $s$, doping concentrations of $\mathrm{n}, \mathrm{p}, \mathrm{n}^{+}$, and $\mathrm{p}^{+}$regions, $N_{\mathrm{A}}, N_{\mathrm{D}}, N_{\mathrm{A}}^{+}$, and $N_{\mathrm{D}}^{+}$, respectively, total width of the $\mathrm{p}$ and $\mathrm{n}$ regions, $W$, width between electrodes, $W^{+}$, and interval $w_{\mathrm{x}}$ and width $w_{\mathrm{y}}$ of the interleaved junction. Values used for these parameters in this experiment are summarized in Table 2. Fundamentally, we used $L=200 \mu \mathrm{m}$ and $n_{\mathrm{g}}=22$ (experimental value shown below) given by the listed values of $a, 2 r$, and $s$ $[16,17]$. In addition, we also prepared $L=90 \mu \mathrm{m}$ samples for comparison. Typical values were employed for $N_{\mathrm{A}}^{+}, N_{\mathrm{D}}^{+}$, and $W^{+}$ [15-17]. We set $W=4 \mu \mathrm{m}$ to avoid the free carrier absorption loss in $\mathrm{p}^{+}$and $\mathrm{n}^{+}$regions [17]. On the other hand, we changed $N_{\mathrm{A}}, N_{\mathrm{D}}, w_{\mathrm{x}}$, and $w_{\mathrm{y}}$ as parameters for investigation.

\section{PHASE SHIFT}

The phase shift $\Delta \phi$ of the waveguide mode in a MZ modulator when the voltage is applied to the $\mathrm{p} / \mathrm{n}$ junction is given by the following:

$$
\Delta \phi=\Delta k L=k_{0} n_{\mathrm{g}} \frac{\Delta n_{\mathrm{eq}}}{n_{\mathrm{eq}}} \zeta L, \quad \zeta \equiv \frac{n_{\mathrm{eq}}}{\omega_{\mathrm{b}}} \frac{d \omega_{\mathrm{b}}}{d n_{\mathrm{eq}}},
$$

where $\Delta k$ is the wavenumber shift of the waveguide mode, $k_{0}=$ $2 \pi / \lambda$ is the wavenumber in vacuum, $n_{\mathrm{eq}}$ is the modal equivalent index, and $\Delta n_{\mathrm{eq}}$ is the change of $n_{\mathrm{eq}}$ with the voltage. For the transverse-electric (TE) polarized light exhibiting a photonic band gap at $\lambda=1.55 \mu \mathrm{m}, n_{\mathrm{g}} \sim 20$ and $n_{\mathrm{eq}}=2.33$ are obtained for $a, 2 r$, and $s$ values in Table 2 . $\zeta$ is almost a constant value close to 1 , where $\omega_{\mathrm{b}}$ is the photonic band frequency. $\Delta \phi$ in Equation (1) becomes twice larger when the push-pull drive is used, which generates the opposite sign of $\Delta n_{\mathrm{eq}}$ to the two phase shifters.

$\Delta n_{\mathrm{eq}}$ is generated by the local index change of $\mathrm{Si}, \Delta n_{\mathrm{Si}}$, due to the carrier plasma effect that occurs when the carrier distribution is changed by applying voltage. We calculated the carrier distribution, as shown in Figure 3, using commercial simulator, Lumerical DEVICE. Here, we assume linear $\mathrm{p} / \mathrm{n}$ junction (device $\# 1$ ) and interleaved one (device \#2 in Table 2) and set middle doping concentrations in Table 2. It is clear that the depletion

Table 2 | Design parameters.

\begin{tabular}{ll}
\hline Parameter & Values \\
\hline$L[\mu \mathrm{m}]$ & 90,200 \\
$a[\mathrm{~nm}]$ & 400 \\
$2 r[\mathrm{~nm}]$ & 220 \\
$s[\mathrm{~nm}]$ & 80 \\
$N_{\mathrm{A}}\left[10^{17} \mathrm{~cm}^{-3}\right]$ & 2.4 (low), 4.8 (middle), 9.5 (high) \\
$N_{\mathrm{D}}\left[10^{17} \mathrm{~cm}^{-3}\right]$ & 1.4 (low), 2.9 (middle), 5.7 (high) \\
$N_{\mathrm{A}}^{+}\left[10^{19} \mathrm{~cm}^{-3}\right]$ & 1 \\
$N_{\mathrm{D}}^{+}\left[10^{19} \mathrm{~cm}^{-3}\right]$ & 1 \\
$W^{-3}[\mu \mathrm{m}]$ & 4 \\
$W^{+}[\mu \mathrm{m}]$ & 21 \\
$W_{x}[\mathrm{~nm}]$ & $-(\# 1), 300$ (\#2, \#3) \\
$W_{y}[\mathrm{~nm}]$ & $-(\# 1), 600$ (\#2), 1000 (\#3) \\
\hline
\end{tabular}

\#1 shows linear p/n junction and \#2 and \#3, interleaved one. 


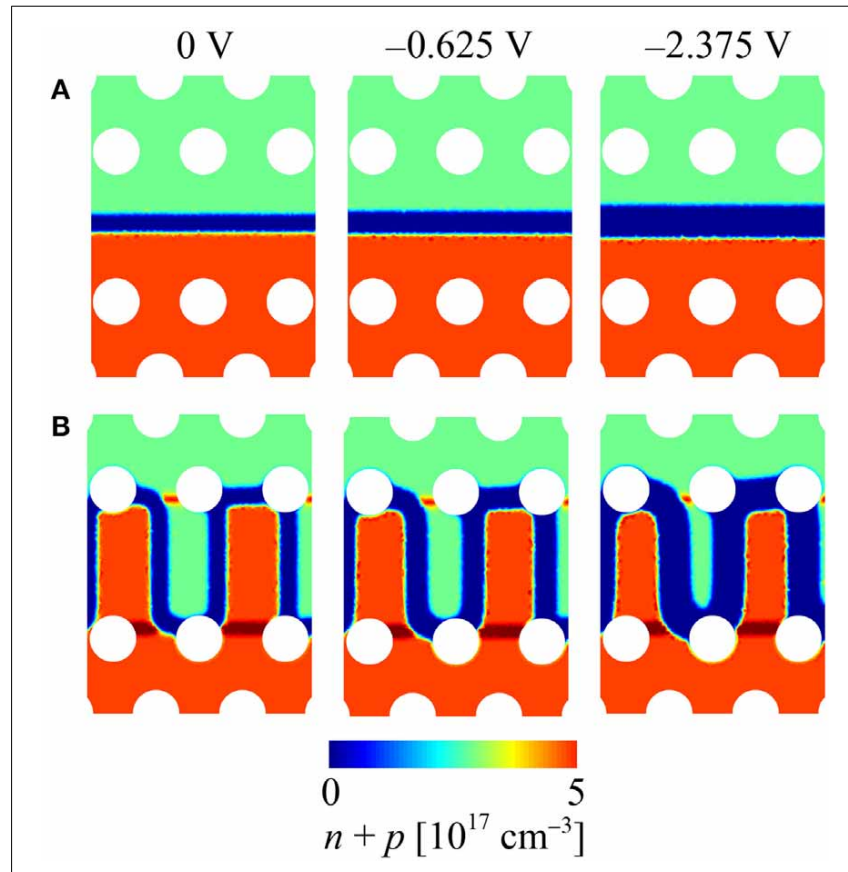

FIGURE 3 | Calculation results of carrier distribution around the center of LSPCW (model I of Figure 2). (A) Device \#1 with linear junction. (B) Device \#2 with interleaved junction. Blue region shows depletion region.

region extends with increasing the reverse bias. Obviously, the interleaved junction produces larger depletion region around the waveguide center. $\Delta n_{\mathrm{Si}}$ can be estimated by the following formula by Soref and Bennett [26]:

$$
\Delta n_{\mathrm{Si}}=-8.8 \times 10^{-22} \Delta n-8.5 \times 10^{-18} \Delta p^{0.8}
$$

When the depletion region extends, both $\Delta n\left[\mathrm{~cm}^{-3}\right]$ and $\Delta p$ $\left[\mathrm{cm}^{-3}\right]$ decrease, leading to a positive $\Delta n_{\mathrm{Si}}$. Figure $4 \mathrm{~A}$ shows the spatial distribution of $\Delta n_{\mathrm{Si}}$ in the unit cell model of LSPCW (II in Figure 2), when the applied voltage is changed from -0.625 to $-2.375 \mathrm{~V}$, which correspond to dc bias voltage $V_{\mathrm{DC}}=-1.50 \mathrm{~V}$ and drive voltage $V_{\mathrm{pp}}=1.75 \mathrm{~V}$ in the modulation experiment. Here is an example for the linear junction corresponding to Figure 3A. It is observed that $\Delta n_{\mathrm{Si}}$ is localized at both edges of the depletion region. Negative $\Delta n_{\mathrm{Si}}$ appeared at some points simply due to numerical imperfection. However, we can neglect it because the number of points exhibiting negative $\Delta n_{\mathrm{Si}}$ points is limited and the absolute values of negative $\Delta n_{\mathrm{Si}}$ are much smaller than positive ones. Figure 4B shows the distribution of the waveguide mode, which is calculated by Lumerical FDTD Solutions. Although this distribution can change with $\Delta n_{\mathrm{Si}}$, it is negligible. The mode is almost localized at the waveguide center, but penetrates into the third rows of airholes. Eventually, the mode in the LSPCW distributes more widely than that in rib waveguide modulator. Finally, $\Delta n_{\mathrm{eq}}$ is given by

$$
\Delta n_{\mathrm{eq}}=\frac{\int \Delta n_{\mathrm{Si}}|E|^{2} d x d y d z}{\int|E|^{2} d x d y d z}
$$

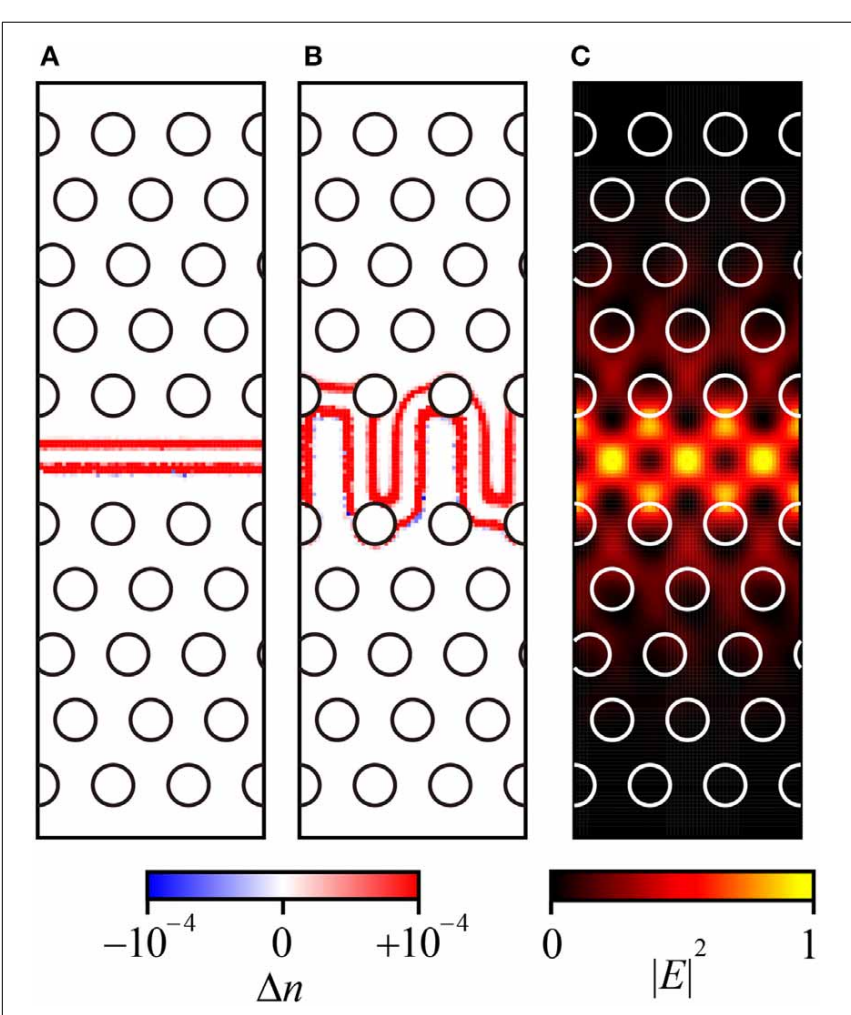

FIGURE 4 | (A,B) Index change of $\mathrm{Si}$ in device \#1 with linear junction and \#2 with interleaved junction ( $w_{y}=600 \mathrm{~nm}$ ), respectively, and (C). Waveguide mode intensity in unit cell model II in Figure 2.

We calculated $\Delta n_{\mathrm{eq}}$ for device \#1 with the linear junction and devices \#2 and \#3 with interleaved junctions with $w_{x}=300 \mathrm{~nm}$ and $w_{y}=600$ or $1000 \mathrm{~nm}$, respectively. The results are summarized in Table 3. Corresponding to the larger depletion region of the interleaved junction \#2 and \#3, $\Delta n_{\mathrm{eq}}$ increases to 1.7 and 2.0 times that of the linear junction, respectively.

Similarly, we calculated $\Delta n_{\mathrm{eq}}$ for a typical rib waveguide modulator with the central linear junction and middle doping. Assuming the width and height of the rib modulator to be 400 and $210 \mathrm{~nm}$, respectively, and the slab thickness to be $60 \mathrm{~nm}, \Delta n_{\mathrm{eq}}$ was obtained as $5.0 \times 10^{-5}$. Since $\Delta n_{\mathrm{eq}}$ in the LSPCW was calculated as $2.1 \times 10^{-5}, \Delta n_{\mathrm{eq}}$ in the LSPCW is 0.42 times that in the rib waveguide. As mentioned above, this is caused mainly by the LSPCW's slow light mode deeply penetrating into the photonic crystal. As $n_{\mathrm{g}}$ of the LSPCW and rib waveguide are 22-24 (measrued values shown later) and 4 , respectively, $n_{\mathrm{g}}$ suggests 5 -fold enhancement. However, the efficiency and the enhancement should be evaluated by the product of $n_{\mathrm{g}}$ and $\Delta n_{\mathrm{eq}}$. as seen in Equation (5). Eventually, the wider mode of the LSPCW reduces the net slow light enhancement to 2.3-2.5.

\section{MODULATION LOSS}

Figure 5 shows $\Delta \phi$ and $M L$ calculated for $L$ using Equations $(4,5)$, assuming the push-pull drive and the same parameters as for Figures 3, 4. Naturally $\Delta \phi$ increases and $M L$ decreases as $L$ gets longer. The interleaved junction is effective for increasing $\Delta \phi$. While $M L$ becomes $<1 \mathrm{~dB}$ at $L$ as large as $439 \mu \mathrm{m}$ 
Table 3 | Comparison of performance between three $\mathrm{p} / \mathrm{n}$ junctions.

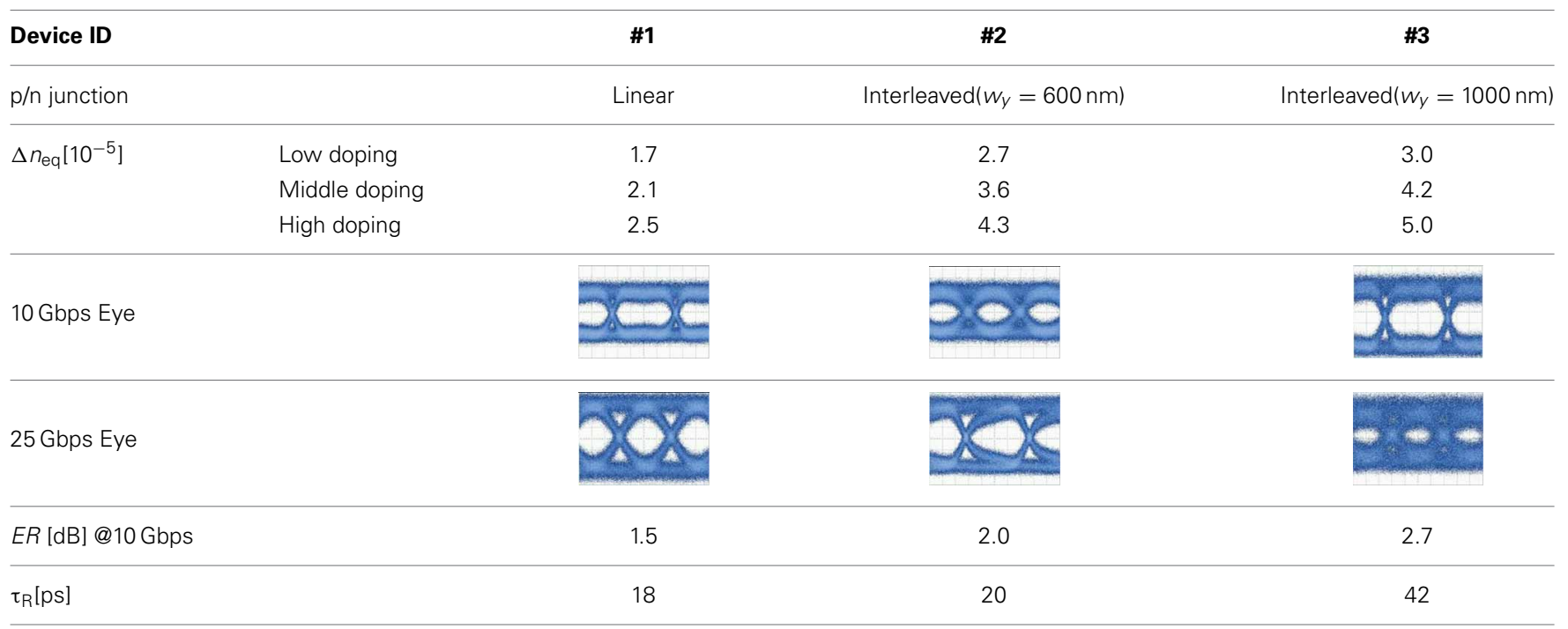

Regarding measurement results discussed later, we used $L=90 \mu \mathrm{m}$ devices with high doping concentration and set $M L=3 \mathrm{~dB}, V_{D C}=-0.90 \mathrm{~V}$ and $V_{p p}=1.75 \mathrm{~V}$. PRBS signal is $2^{31}-1$ bits. $\tau_{\mathrm{R}}$ of the measurement setup is $16 \mathrm{ps}$.

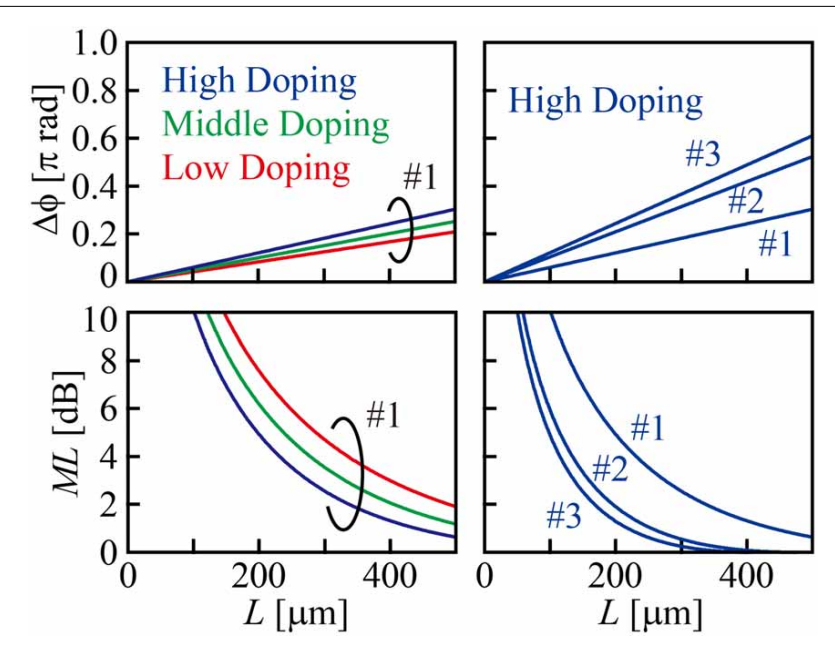

FIGURE 5 | Calculation results of phase shift and modulation loss with phase shifter length for device \#1-\#3 when $E R=\mathbf{3} \mathrm{dB}$ is assumed. Voltage change is set from -0.625 to $-2.375 \mathrm{~V}$.

with the linear junction, it does at $L=255 \mu \mathrm{m}$ with the interleaved junction $\left(w_{y}=600 \mathrm{~nm}\right)$ and at $L=219 \mu \mathrm{m}$ with that $\left(w_{y}=1000 \mathrm{~nm}\right)$.

\section{RC TIME CONSTANTS}

When the electrical resistance and capacitance at electrodes and additional parasitic capacitance are negligible, the operation speed of the modulator is dominated by the $R C$ time constant $\tau_{\mathrm{RC}}$. The resistance $R$ around the $\mathrm{p} / \mathrm{n}$ junction can be evaluated from the current for the applied voltage. We calculated it using DEVICE, assuming the same parameters as for Figure 3 and $L=200 \mu \mathrm{m}$. Then we obtained resistances for $\mathrm{p}$ and $\mathrm{n}$ regions to be 58 and $38 \Omega$, respectively, resulting in $R=96 \Omega$. This value does not depend much on the junction profile and is determined mainly by the total width of the $\mathrm{p}$ and $\mathrm{n}$ regions, $W$. Since we fixed $W=4 \mu \mathrm{m}$ to avoid the absorption loss as mentioned above, $R$ is hardly reduced even after the fine adjustment of $W$. The reduction is rather expected by increasing doping concentrations. This $R$ is 3-6 times larger than rib waveguide modulators. This is caused by the wide $W$ originating from the deep field penetration, which is a drawback of slow light.

The junction capacitance $C$ can be evaluated by the relation between the charge accumulated at the junction and the applied voltage $V$. Figure 6 shows the calculation results of $C, \tau_{\mathrm{RC}}$, and 3 -dB cutoff frequency $f_{3 \mathrm{~dB}}$. For device $\# 1$ with the linear junction, $C=26-50 \mathrm{fF}$ and $f_{3 \mathrm{~dB}}=33-65 \mathrm{GHz}$ are estimated for $V$ from $0 \mathrm{~V}$ to $-3 \mathrm{~V}$. $f_{3 \mathrm{~dB}}$ increases as more $V$ is applied, while $d C / d V$ decreases, meaning that $\Delta \phi$ diminishes. $C$ becomes twice for \#2 with the interleaved junction $\left(w_{y}=600 \mathrm{~nm}\right)$ and 2.5-3.1 times for \#3 with that $\left(w_{y}=1000 \mathrm{~nm}\right)$, and the voltage dependence of $C$ increases proportionally. This corresponds the enhancement of $\Delta \phi$ shown in Section Phase Shift and simultaneously means the reduced bandwidth. Therefore, the optimum voltage and $w_{y}$ exist for a target bandwidth. If we design toward $f_{3 \mathrm{~dB}}=$ $25 \mathrm{GHz}$, for example, a bias voltage of around $-1.5 \mathrm{~V}$ is optimum for \#2.

Now, the time constant does not depend on $L$ because $R$ is inversely proportional to and $C$ is proportional to $L$. Hence, $\tau_{\mathrm{RC}}$ and $f_{3 \mathrm{~dB}}$ similar to those in Figure 6 may be obtainable at different L. Actually, however, the internal resistance of $50 \Omega$ in the modulator driver is included in the whole circuit. On this condition, longer $L$ is not effective for reducing $R$, thus rather reduces $f_{3 \mathrm{~dB}}$. Shorter $L$ is more advantageous concerning $f_{3 \mathrm{~dB}}$, but the decrease in $C$ results in smaller $\Delta \phi$ and lower $E R$. Therefore, the careful optimization and balancing are necessary. Anyway, the internal resistance reduces $f_{3 \mathrm{~dB}}$ at any $L$ than that in Figure 6 (for example, $\sim 0.66$ times at $L=200 \mu \mathrm{m}$ ). Still 25 Gbps modulation will be possible by employing \#1 and \#2. For longer $L$, the phase matching between slow light and rf 


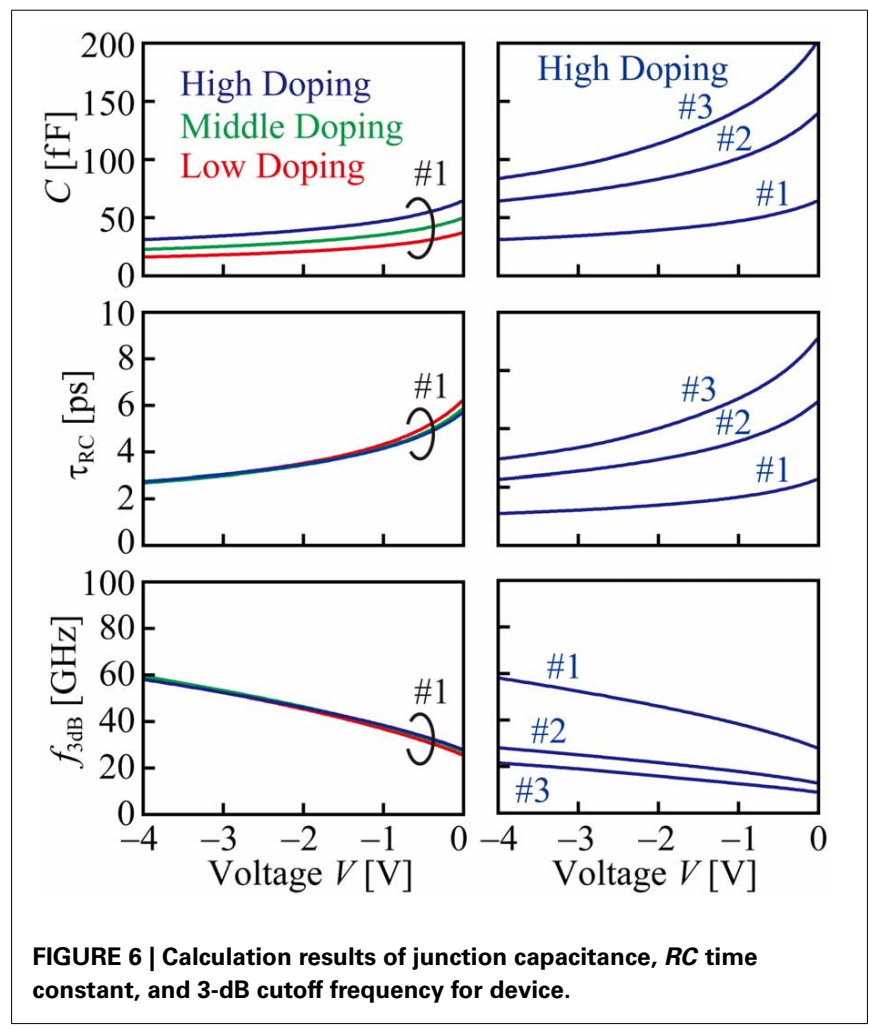

signal will be another issue, which we will discuss at another instance.

\section{FABRICATION AND MEASUREMENT}

For fabrication, we used the foundry service using CMOScompatible process (minimum linewidth of $180 \mathrm{~nm}$ ) provided by Institute of Microelectronics, Singapore. Figure 7 shows the fabricated device. The basic structure is the same as that in Figure 2A. $\mathrm{p}$ and $\mathrm{n}$ regions were formed by boron and phosphorus ion implantations, respectively. As shown in Table 2, three types of doping concentrations were prepared. Electrodes of the phase shifter and phase tuner were 700-nm-thick Al. GSGSG rf probe pad was arranged on the input side, while the termination on the output side was omitted. The phase tuner was shortened to 36.5 from $200 \mu \mathrm{m}$ in previous studies [16, 17], considering the slow light enhancement. Thermal isolation trenches of more than $50 \mu \mathrm{m}$ depth were formed around the phase tuner. Figure 8 shows the transmission intensity and $n_{\mathrm{g}}$ spectra of the device. The latter was measured by modulation phase shift method. Low-dispersion slow light exhibiting $n_{\mathrm{g}}=22-24$ and $\Delta \lambda \sim 14 \mathrm{~nm}$ was obtained. Although these spectra were measured for interleaved junction (\#2) and high doping concentrations, similar spectra were obtained for other devices. The insertion loss at the transmission peak intensity, estimated by comparing the output intensity with that from the Si wire waveguide of the same length was less than $5 \mathrm{~dB}$, which is lower than $7 \mathrm{~dB}$ in the previous studies. It is attributed to the further optimization and loss reduction at the splitter of the $M Z$ circuit and connection between the Si wire and LSPCW. Black circles in Figure 1 show the measured output intensity at each operating
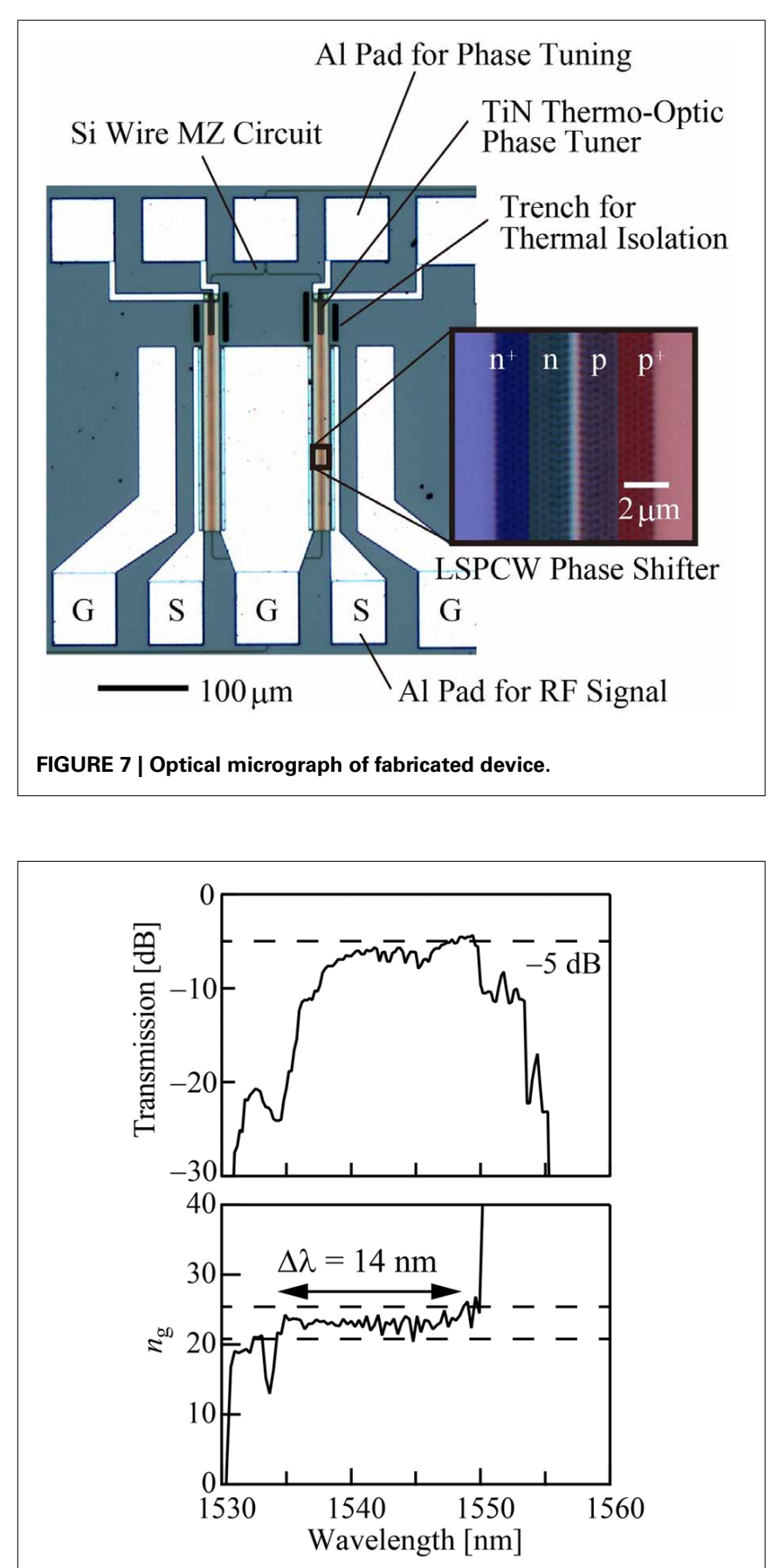

FIGURE 8 | Measured spectra of transmission intensity and group index. The broken line shows the wavelength which was used in the modulation experiment.

point changed by the heater power for this device. The circles are well fitted to the theoretical curve when a $0.02 \pi$ offset is assumed. This indicates that phase shift is proportional to heater power. This offset and the bottom intensity saturated at $-25 \mathrm{~dB}$ around $\phi=\pi$ suggest that a slight difference between two optical paths was induced by fabrication errors, although it is a trivial problem. 
In the modulation experiment, we directly applied the same dc bias $V_{\mathrm{DC}}$ to the two phase shifters and opposite sign of pseudo-random bit sequence (PRBS) non-return-to-zero (NRZ) signal $V_{\mathrm{pp}}$ (push-pull drive) by using pulse pattern generator Anritsu MG3692B, MP181020A, MP181040A and GSGSG RF probe with a $40 \mathrm{GHz}$ bandwidth. Compared with previous studies, $\mathrm{S} / \mathrm{N}$ was improved by removing electrical amplifiers and bias tee and shortening rf cables. Input laser light was set at $1555.5 \mathrm{~nm}$, which is the center wavelength of the spectra in Figure 8. It was adjusted to TE by polarization controller, and coupled from lensed fiber to Si inverse-tapered spot size converter, which was integrated on a chip. Modulated light was similarly coupled to another fiber and detected by optical sampling oscilloscope Agilent 86100C, 86109A.

The following measurement results used $V_{\mathrm{DC}}=-1.5 \mathrm{~V}$, $V_{\mathrm{pp}}=1.75 \mathrm{~V}$, and $B R=25 \mathrm{Gbps}$ as base settings. Figure 9 shows 25 Gbps eye patterns at each operating point in Figure 1. When $\phi$ is close to 0 (point A), $M L$ and $E R$ are small, resulting in closed eye. When $\phi$ is close to $\pi$ (point $\mathrm{E}$ ), ER becomes large, and S/N is degraded by the large $M L$. Figure 10 summarizes the dependence on $B R, V_{\mathrm{pp}}$, and $V_{\mathrm{DC}}$ at point $\mathrm{D}$. In $\mathrm{A}$, clear open eye with $E R \sim 4 \mathrm{~dB}$ is maintained in the range of $10-25$ Gbps. As seen in Figure 6, $f_{3 \mathrm{~dB}}$ for device \#1 with the linear junction is sufficiently large. Therefore, higher-speed operation will be confirmed if higher speed measurement setup is available. In $\mathrm{B}, E R$ is larger at higher $V_{\mathrm{pp}}$ and $E R=3 \mathrm{~dB}$ is obtained at $V_{\mathrm{pp}}=1.5 \mathrm{~V}$. In C, $E R$ is increased as $V_{\mathrm{DC}}$ approaches to $0 \mathrm{~V}$. Although small $V_{\mathrm{DC}}$ generally increases $C$ and decrease the response speed as seen in Figure 6, its influence did not appear because of the sufficient bandwidth of the linear junction.

In Figure 10, we can observe clear eye patterns from low voltages, but still $\Delta \phi$ is small and $M L$ is as large as $7 \mathrm{~dB}$. When employing the interleaved junction, $\Delta \phi$ increases although the response is degraded. Table 3 compares eye patterns, ER and 20$80 \%$ rise time $\tau_{R}$ for $\# 1$ with the linear junction and \#2 and \#3 with interleaved junctions. Due to the limited samples, the devices shown here are all highly doped and have a length of $L=90 \mu \mathrm{m}$. We set $M L=3 \mathrm{~dB}$ for moderate eye opening; $E R$ is small but we

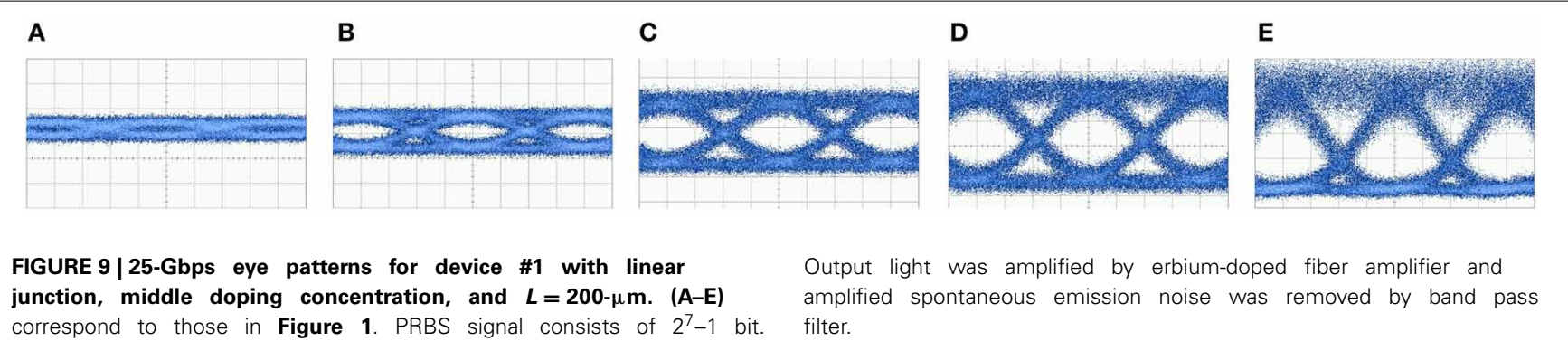

A

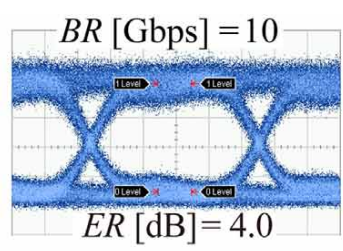

B

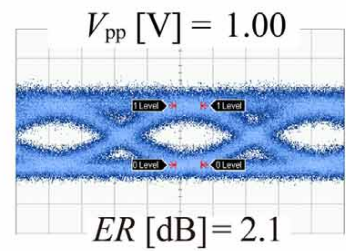

C

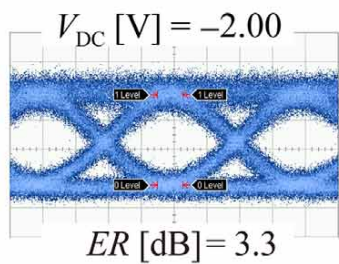

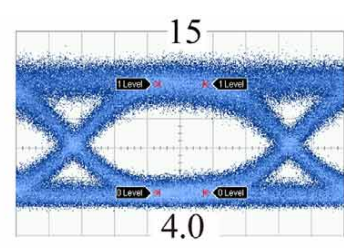

1.25

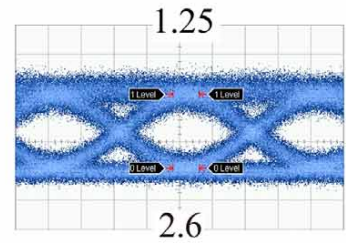

$-1.50$

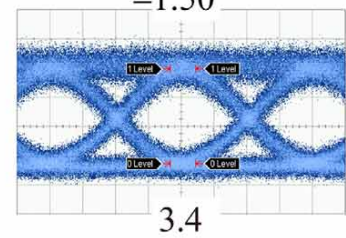

3.4

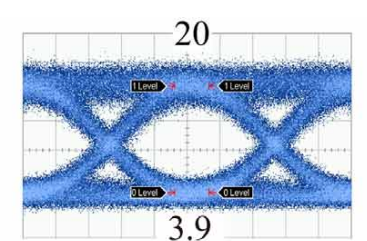

3.9

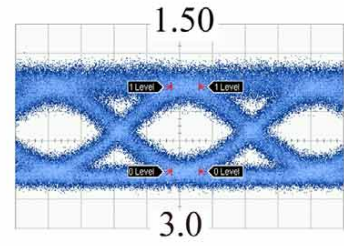

$-1.00$

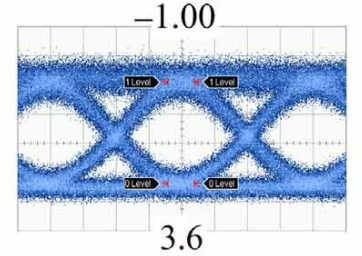

FIGURE 10 | Parameter dependence of the eye pattern for device \#1 with liner junction and middle doping concentration. Basic settings, and measurement condition are the same as those for
Figure 9, and operating point was set at $D(M L=7 \mathrm{~dB})$ in

Figure 1. (A) $B R$ dependence. (B) $V_{p p}$ dependence. (C) $V_{D C}$ dependence.
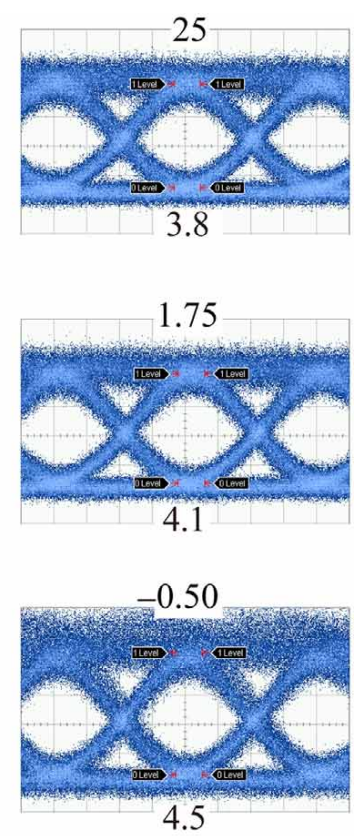
can compare them qualitatively. $w_{x}=200 \mathrm{~nm}$ seems to be better because the interval $2 w_{x}$ is the same as $a$, but actually we observed more closed eye for $w_{\mathrm{x}}=200 \mathrm{~nm}$ and lower $E R$, which might be caused by the $\mathrm{p} / \mathrm{n}$ compensation. Although $E R$ became maximum at $w_{x}=300 \mathrm{~nm}$ and $w_{y}=1000 \mathrm{~nm}$, the response was slow, as seen from $\tau_{\mathrm{R}}=42 \mathrm{ps}$ and $\mathrm{S} / \mathrm{N}$ is particularly degraded at $25 \mathrm{Gbps}$, which might be caused by the pattern effect. Moderate ER and $\tau_{\mathrm{R}}$ were obtained and clear $25 \mathrm{Gbps}$ open eye was observed at $w_{x}=300 \mathrm{~nm}$ and $w_{y}=600 \mathrm{~nm}$.

Figure 11 shows the dependence of $E R$ on the doping concentration in the $L=90 \mu \mathrm{m}$ device $\# 1$ and \#2. ER increased at a higher doping concentration for both junctions, and the increase is particularly large in the interleaved junction. The decrease in the transmission intensity, which could occur due to the free carrier absorption, was less than $1 \mathrm{~dB}$ even at the high doping concentration. Figure 12 shows 25 Gbps eye pattern in the

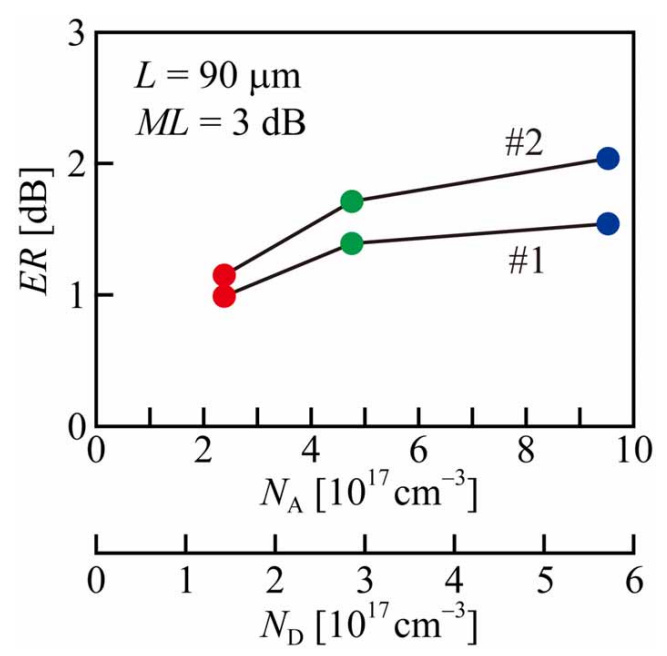

FIGURE 11 | Doping concentration of $E R$ at $10-$ Gbps $\left(2^{31}-1\right.$ bits) for device \#1 with linear junction and device \#2 with interleaved junction $\left(w_{x}=300 \mathrm{~nm}, w_{y}=600 \mathrm{~nm}\right)$.

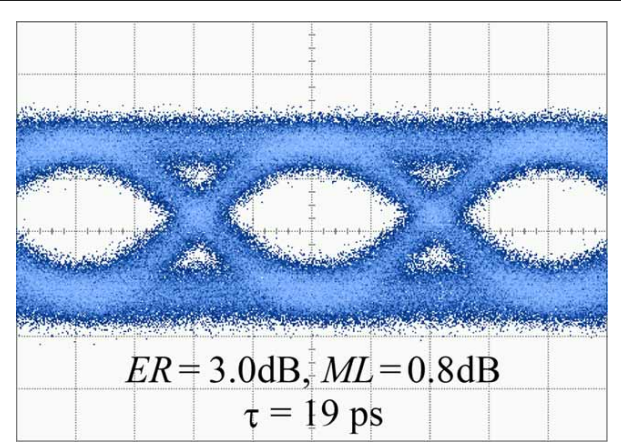

FIGURE 12 | ( $2^{31}-1$ bits) eye pattern for $L=200 \mu \mathrm{m}$ device \#2 with interleaved junction $\left(w_{\boldsymbol{y}}=\mathbf{6 0 0} \mathrm{nm}\right)$ and high doping concentration. $V_{\mathrm{pp}}=1.75 \mathrm{~V}$ and $V_{\mathrm{DC}}=-0.90 \mathrm{~V}$. In this measurement, higher-speed pulse pattern generator (Anritsu 183021A; the measurement $\tau_{R}$ is $11 \mathrm{ps}$ ) was used.
$L=200 \mu \mathrm{m}$ device with the interleaved junction and high doping concentration. We observed $E R=3.0 \mathrm{~dB}$ simultaneously with $M L=0.8 \mathrm{~dB}$. We observed $\tau_{\mathrm{R}}$, as short as $19 \mathrm{ps}$, which indicates higher speed modulation.

\section{CONCLUSIONS}

We discussed the index and phase changes in Si LSPCW MZ modulators from theoretical calculations and experiment and estimated that the advantage of slow light is 2.3-2.5 times compared with rib waveguide modulators and that additional enhancement of 1.7-2.0 times is obtainable by employing the interleaved junction and 1.2 times by increasing the doping concentration. Increasing the phase shift lowers the modulation loss. We also discussed the response speed and concluded that the linear junction has a sufficient modulation bandwidth of $f_{3 \mathrm{~dB}}=33-65 \mathrm{GHz}$ and that it decreases to less than half when employing interleaved junctions. We experimentally obtained $25 \mathrm{Gbps}$ clear open eyes with extinction ratios of 3.0-4.1 dB at low voltages of $V_{\mathrm{pp}}=1.50$ $1.75 \mathrm{~V}$ for the $L=200 \mu \mathrm{m}$ device when $7 \mathrm{~dB}$ modulation loss is added. We further obtained the extinction ratio of $3.0 \mathrm{~dB}$ even with a modulation loss of $0.8 \mathrm{~dB}$ by using optimum interleaved junction and high doping concentration. This performance meets most of the requirements for current and near-future optical interconnects.

\section{ACKNOWLEDGMENT}

This study was partly supported by New Energy and Industrial Technology Development Organization.

\section{REFERENCES}

1. Reed GT, Mashanovich G, Gardes FY, Thomson DJ. Silicon optical modulators. Nat Photonics (2010) 4:518. doi: 10.1038/nphoton.2010.179

2. Zhou G, Geis MW, Spector SJ, Gan F, Grein ME, Schulein RT, et al. Effect of carrier lifetime on forward-biased silicon Mach-Zehnder modulators. Opt Express (2008) 21:11869. doi: 10.1364/OE.16.005218

3. Akiyama S, Imai M, Baba T, Akagawa T, Hirayama N, Noguchi $Y$, et al. Compact PIN-diode-based silicon modulator using side-wall-grating waveguide. IEEE J Sel Top Quantum Electron. (2014) 19:3401611. doi: 10.1109/JSTQE.2013.2278438

4. Baba T, Akiyama S, Imai M, Hirayama N, Takahashi H, Noguchi Y, et al. 50$\mathrm{Gb} / \mathrm{s}$ ring-resonator-based silicon modulator. Opt Express (2013) 21:11869. doi: 10.1364/OE.21.011869

5. Nguyen HC, Sakai Y, Shinkawa M, Ishikura N, Baba T. $10 \mathrm{~Gb} / \mathrm{s}$ operation of photonic crystal silicon optical modulators. Opt Express (2012) 19:13000. doi: 10.1364/OE.19.013000

6. Timurdogan E, Sorace-Agaskar CM, Sun J, Hosseini S, Biberman A, Watts MR. An ultralow power athermal silicon modulator. Nat Commun. (2014) 5:4008 doi: $10.1038 /$ ncomms5008

7. Xiao X, Xu H, Li X, Hu Y, Xiong K, Li Z, et al. 25 Gbit/s silicon microring modulator based on misalignment-tolerant interleaved $\mathrm{PN}$ junctions. Opt Express (2012) 20:2507. doi: 10.1364/OE.20.002507

8. Ding R, Liu Y, Li Q, Yang Y, Ma Y, Padmaraju K, et al. Design and characterization of a 30-GHz bandwidth low-power silicon traveling-wave modulator. Opt Commun. (2014) 321:124. doi: 10.1016/j.optcom.2014.01.071

9. Yang L, Ding J. High-speed silicon Mach-Zehnder optical modulator with large optical bandwidth. J Lightwave Technol. (2014) 32:966. doi: 10.1109/JLT.2013.2295401

10. Xu H, Li X, Xiao X, Li Z, Yu Y, Yu J. Demonstration and characterization of high-speed silicon depletion-mode Mach-Zehnder modulators. IEEE J Sel Top Quantum Electron. (2014) 20:3400110. doi: 10.1109/JSTQE.2013.2293763

11. Kim G, Park JW, Kim IG, Kim S, Jang K, Kim SA, et al. Compact-sized highmodulation-efficiency silicon Mach-Zehnder modulator based on a vertically 
dipped depletion junction phase shifter for chip-level integration. Opt Lett. (2014) 39:2310. doi: 10.1364/OL.39.002310

12. Marris-Morini D, Baudot C, Fédéli JM, Rasigade G, Vulliet N, Souhaité A, et al. Low loss $40 \mathrm{Gbit} / \mathrm{s}$ silicon modulator based on interleaved junctions and fabricated on $300 \mathrm{~mm}$ SOI wafers. Opt Express (2013) 21:22471. doi: 10.1364/OE.21.022471

13. Xiao X, Xu H, Li X, Li Z, Chu T, Yu Y, et al. High-speed, low-loss silicon MachZehnder modulators with doping optimization. Opt Express (2013) 21:4116. doi: 10.1364/OE.21.004116

14. Thomson DJ, Gardes FY, Fedeli J, Zlatanovic S, Hu Y, Kuo BPP, et al. 50$\mathrm{Gb} / \mathrm{s}$ silicon optical modulator. IEEE Photonics Technol Lett. (2012) 24:234. doi: 10.1109/LPT.2011.2177081

15. Nguyen HC, Sakai Y, Shinkawa M, Ishikura N, Baba T. Photonic crystal silicon optical modulators: carrier-injection and depletion at $10 \mathrm{~Gb} / \mathrm{s}$. IEEE J Quantum Electron. (2012) 48:210. doi: 10.1109/JQE.2011.2174338

16. Nguyen HC, Hashimoto S, Shinkawa M, Baba T. Compact and fast photonic crystal silicon optical modulators. Opt Express (2012) 20:22465. doi: 10.1364/OE.20.022465

17. Nguyen HC, Yazawa N, Hashimoto S, Otsuka S, Baba T. Sub-100 $\mu \mathrm{m}$ photonic crystal Si optical modulators: spectral, athermal and high-speed performance. IEEE J Sel Top Quantum Electron. (2013) 19:3400811. doi: 10.1109/JSTQE.2013.2265193

18. Xu H, Xiao X, Li X, Hu Y, Li Z, Chu T, et al. High speed silicon Mach-Zehnder modulator based on interleaved PN junctions. Opt Express (2012) 20:15093. doi: 10.1364/OE.20.015093

19. Yu H, Pantouvaki M, Campenhout JV, Korn D, Komorowska K, Dumon P, et al. Performance tradeoff between lateral and interdigitated doping patterns for high speed carrier-depletion based silicon modulators. Opt Express (2012) 20:12926. doi: 10.1364/OE.20.012926

20. Kim Y, Takenaka M, Osada T, Hata M, Takagi S. Strain-induced enhancement of plasma dispersion effect and free-carrier absorption in SiGe optical modulators. Sci Rep. (2014) 4:4683. doi: 10.1038/srep04683

21. Liu J, Beals M, Pomerene A, Bernardis S, Rong S, Cheng J, et al. Waveguideintegrated, ultra-energy GeSi electro-absorption modulators. Nat Photonics (2008) 2:433. doi: 10.1038/nphoton.2008.99
22. Baba T. Slow light in photonic crystals. Nat Photonics (2008) 2:465. doi: 10.1038/nphoton.2008.146

23. Baba T, Nguyen HC, Ishikura N, Suzuki K, Shinkawa M, Hayakawa R, et al. Photonic crystal slow light devices fabricated by CMOS-compatible process. IEICE Electron Express (2013) 10:1. doi: 10.1587/elex.10.201 32002

24. Hamachi Y, Kubo S, Baba T. Slow light with low dispersion and nonlinear enhancement in a lattice-shifted photonic crystal waveguide. Opt Lett. (2009) 34:1072. doi: 10.1364/OL.34.001072

25. Li Z, Xu D, McKinnon WR, Janz S, Schmid JH, Cheben P, et al. Silicon waveguide modulator based on carrier depletion in periodically interleaved PN junctions. Opt Express (2009) 17:15947. doi: 10.1364/OE.17. 015947

26. Soref RA, Bennett BR. Electrooptical effects in silicon. IEEE J Quantum Electron. (1987) QE-23:123. doi: 10.1109/JQE.1987.1073206

Conflict of Interest Statement: The authors declare that the research was conducted in the absence of any commercial or financial relationships that could be construed as a potential conflict of interest.

Received: 02 September 2014; accepted: 13 October 2014; published online: 06 November 2014.

Citation: Terada Y, Ito H, Nguyen HC and Baba T (2014) Theoretical and experimental investigation of low-volgage and low-loss 25-Gbps Si photonic crystal slow light Mach-Zehnder modulators with interleaved p/n junction. Front. Phys. 2:61. doi: 10.3389/fphy.2014.00061

This article was submitted to Interdisciplinary Physics, a section of the journal Frontiers in Physics.

Copyright (c) 2014 Terada, Ito, Nguyen and Baba. This is an open-access article distributed under the terms of the Creative Commons Attribution License (CC BY). The use, distribution or reproduction in other forums is permitted, provided the original author(s) or licensor are credited and that the original publication in this journal is cited, in accordance with accepted academic practice. No use, distribution or reproduction is permitted which does not comply with these terms. 